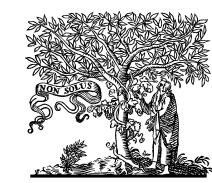

ELSEVIER MASSON
Elsevier Masson France
NUTRITION CLINIQUE et MÉTABOLISME

Nutrition clinique et métabolisme 27 (2013) 82-86

Revue générale

\title{
Obésité et carences préopératoires
}

\section{Obesity and pre-operative nutritional deficiencies}

\author{
Jenny De Flines ${ }^{a}$, Laurent Bruwier ${ }^{\mathrm{a}}$, Arnaud DeRoover ${ }^{\mathrm{b}}$, Nicolas Paquot ${ }^{\mathrm{a}, *}$ \\ ${ }^{a}$ Service de diabétologie, nutrition et maladies métaboliques, département de médecine, CHU Sart-Tilman, 4000 Liège 1, Belgique \\ ${ }^{\mathrm{b}}$ Service de chirurgie digestive, CHU Sart-Tilman, 4000 Liège 1, Belgique \\ Disponible sur Internet le 16 janvier 2013
}

\section{Résumé}

Les carences nutritionnelles sont considérées comme rares au sein de la population occidentale compte tenu de la pléthore alimentaire actuelle. Cependant, en raison d'une alimentation riche en énergie mais pauvre du point de vue nutritionnel (pauvre en protéines, vitamines, minéraux et fibres), des déficiences nutritionnelles en vitamines et minéraux sont fréquemment rapportées chez le sujet obèse. De plus, la chirurgie bariatrique constitue actuellement le traitement le plus efficace de l'obésité morbide, mais une carence en micronutriments représente un problème important dans les suites de la chirurgie, contribuant dès lors à aggraver les carences préexistantes. Dans ce travail, nous envisageons la prévalence des carences nutritionnelles du sujet avec obésité morbide avant chirurgie bariatrique, les conséquences cliniques de ces déficiences et les recommandations pratiques qu'il convient d'appliquer chez ce type de patients.

(C) 2013 Publié par Elsevier Masson SAS.

Mots clés : Micronutriments ; Chirurgie bariatrique ; Déficiences nutritionnelles

\begin{abstract}
It is a common belief that nutritional deficiencies are rare in the Western world due to a wide variety of food supply. However, obese people usually consume dense-energy food but of poor nutritional value that lacks proteins, vitamins, minerals and fiber; consequently, a high prevalence of micronutrient deficiencies in obese subjects has been reported. Moreover, bariatric surgery has been proven the most effective treatment of morbid obesity, but micronutrient deficiency following bariatric surgery is a major concern, worsening pre-operative nutritional deficiencies. In this article, we reviewed the litterature and highlighted the prevalence of nutritional deficiencies in the morbidly obese population prior to bariatric surgery, clinical consequences of these deficiencies and practical recommendations for these subjects.
\end{abstract}

(C) 2013 Published by Elsevier Masson SAS.

Keywords: Micronutrient; Bariatric surgery; Nutritional deficiencies

\section{Introduction}

L'obésité morbide, définie par un indice de masse corporelle (IMC) supérieur à $40 \mathrm{~kg} / \mathrm{m}^{2}$ (ou $\geq$ à $35 \mathrm{~kg} / \mathrm{m}^{2}$ avec la présence de comorbidités en relation avec l'obésité comme le diabète de type 2) constitue un problème majeur de santé publique, car elle altère la qualité de vie [1] et favorise la survenue d'autres affections comme l'hypertension artérielle, l'hypercholestérolémie, les maladies cardiovasculaires, le diabète de type 2 ou encore

\footnotetext{
* Auteur correspondant.

Adresse e-mail : Nicolas.paquot@ulg.ac.be (N. Paquot).
}

certains cancers $[2,3]$. On estime que les sujets avec un IMC entre 40 et $45 \mathrm{~kg} / \mathrm{m}^{2}$ ont une espérance de vie diminuée de huit à dix ans (ce qui est comparable aux effets du tabac) [4]. De plus, c'est surtout la prévalence de l'obésité sévère (IMC supérieur à 40 voire $50 \mathrm{~kg} / \mathrm{m}^{2}$ ), associée aux comorbidités les plus sévères, qui augmente à l'heure actuelle de façon la plus importante dans la population adulte aux États-Unis [5]. Compte tenu des résultats décevants obtenus par les approches non chirurgicales, la chirurgie de l'obésité ou chirurgie bariatrique représente actuellement pour les sujets avec obésité morbide une modalité thérapeutique majeure qui a connu un essor considérable au cours de ces dernières années. Toujours aux États-Unis, le 
nombre de patients opérés est passé de 20000 en 1992 à plus de 100000 en 2003 ; de nombreux travaux ont bien établi l'intérêt de ces techniques, non seulement sur la perte de poids substantielle même à long terme, mais également sur l'amélioration de la qualité de vie [6] et la réduction significative du risque de morbi-mortalité $[7,8]$. Par ailleurs, il est maintenant bien établi qu'un suivi nutritionnel spécifique et rigoureux doit être réalisé chez les patients ayant bénéficié d'une chirurgie de l'obésité, afin d'éviter l'apparition de carences nutritionnelles à moyen et long terme [9]. Plus récemment, l'attention a été attirée sur la situation nutritionnelle des sujets présentant une obésité morbide. En effet, en dépit d'un apport calorique excessif, la faible qualité nutritionnelle des aliments ingérés - à forte teneur calorique mais à pauvre valeur nutritionnelle (manque de protéines, de vitamines, de minéraux et de fibres) - conduit bon nombre de sujets avec obésité morbide à présenter des carences nutritionnelles avant l'intervention chirurgicale [10,11], suggérant que les déficiences nutritionnelles observées en postopératoire ne sont pas seulement le résultat de la malabsorption induite par la chirurgie et la perte de poids rapide, mais sont aussi le résultat d'une forme préexistante de malnutrition.

Dans cette revue, nous analysons quelles sont les carences nutritionnelles préopératoires du sujet obèse les plus fréquemment observées, leur prévalence, leur impact clinique potentiel et le type de prise en charge qui pourrait être proposé.

\section{Carences nutritionnelles chez le sujet avec obésité morbide}

Au cours de ces dernières années, différents travaux ont été publiés visant à cerner la prévalence des carences nutritionnelles chez le sujet avec obésité morbide, ces études ayant été pour la plupart menées chez des sujets qui devaient bénéficier d'une chirurgie bariatrique. Toutefois, la plupart de ces études ont été effectuées de manière rétrospective, parfois sans groupe témoin, rendant l'interprétation des résultats malaisée. Les déficiences sont toujours estimées au travers du dosage plasmatique, ce qui ne constitue pas toujours un bon reflet de l'état exact des réserves et les résultats obtenus sont de plus très variables d'une étude à l' autre. Enfin, les conséquences cliniques de ces carences nutritionnelles, souvent a minima, demeurent mal connues et il n'existe actuellement aucune étude rapportant les résultats d'une supplémentation nutritionnelle préopératoire chez le sujet avec obésité morbide.

\subsection{Prévalence des carences nutritionnelles}

Deux revues de la littérature publiées en 2008 avaient pour but de déterminer la prévalence des déficiences nutritionnelles en vitamines [10] et minéraux [11] chez le sujet obèse. Dans ces deux travaux, toutes les études publiées ont été prises en compte, sans aucune restriction, et les résultats doivent dès lors être interprétés avec une certaine précaution. En ce qui concerne les vitamines, des déficits en vitamine B1 (environ $15 \%$ des sujets) et en vitamine $\mathrm{D}(40 \%)$ sont régulièrement observés. Concernant les minéraux, l'attention était portée sur des déficiences assez fréquentes en sélénium (près de $60 \%$ des sujets avant chirurgie bariatrique), en fer (15\%) et en zinc (entre 20 et $30 \%$ ). Une autre étude, prospective et monocentrique, menée chez 232 sujets obèses (IMC $\geq 35 \mathrm{~kg} / \mathrm{m}^{2}$ ) étudiés consécutivement avant chirurgie bariatrique, démontre principalement une déficience en zinc ( $25 \%)$, en vitamine B12 (18\%) et une carence sévère en vitamine $\mathrm{D}(25 \%)$ avec hyperparathyroïdie secondaire [12]. Près de $50 \%$ des sujets présentaient au moins une de ces trois déficiences. Concernant la vitamine $\mathrm{D}$, on sait que la déficience $(12-20 \mathrm{ng} / \mathrm{mL})$ et même la carence $(<12 \mathrm{ng} / \mathrm{mL})$ sont hautement prévalentes chez les sujets obèses, suite probablement à un stockage dans le tissu adipeux (bien que la relation entre l'importance de la déficience et l'IMC ne soit pas constamment retrouvée). Cette importante prévalence de la déficience en vitamine $\mathrm{D}$ impose une supplémentation rigoureuse après chirurgie, mais un dépistage préopératoire est également souhaitable compte tenu que ce déficit précède le plus souvent la chirurgie bariatrique.

Une autre étude prospective menée chez des sujets adressés pour prise en charge d'une obésité morbide a comparé le niveau plasmatique de différentes vitamines avec un groupe de sujets sains. On retrouve chez 11 à $38 \%$ des sujets obèses une déficience en vitamine $\mathrm{B} 6, \mathrm{C}, \mathrm{D}$ et $\mathrm{E}$, l'importance de la déficience étant en relation avec la sévérité de l'obésité [13]. Schweiger et al. [14] ont tenté d'identifier chez des sujets avec obésité morbide avant chirurgie bariatrique les relations pouvant exister entre les déficiences nutritionnelles et le statut socio-économique et les comorbidités. Aucune relation entre les déficiences nutritionnelles et le niveau d'éducation, le revenu ou la présence de comorbidités (diabète, hypertension, dyslipidémies ou stéatose hépatique) n'a cependant été mise en évidence. Enfin, une étude récente démontre chez des femmes obèses avant chirurgie bariatrique l'importante prévalence de la carence en cuivre $(68 \%)$ et en zinc (74\%) [15]. Le Tableau 1 résume les principales déficiences nutritionnelles rapportées chez les sujets avec obésité morbide avant chirurgie bariatrique.

En collaboration avec le service de chirurgie digestive du CHU de Liège, nous avons analysé la prévalence des principales déficiences nutritionnelles chez 113 patients devant bénéficier d'une chirurgie bariatrique (Tableau 2). Nous retrouvons des

\section{Tableau}

Prévalence des principales déficiences nutritionnelles (\%) observées avant chirurgie bariatrique chez des individus présentant une obésité morbide (les chiffres entre parenthèses se rapportent aux références bibliographiques).

\begin{tabular}{ll}
\hline Concentration plasmatique & $\begin{array}{l}\text { Prévalence de résultats } \\
\text { anormaux, en } \%\end{array}$ \\
\hline Vitamine B1 & $15[10]$ \\
Vitamine B12 & 3 à $18[10,14,15]$ \\
Acide folique & 2 à $25[10,12,14,15]$ \\
Vitamine D & 25 à $70[10,12,15]$ \\
Fer & 14 à $35[11,14]$ \\
Ferritine & 5 à $24[11,12,14,15]$ \\
Hémoglobine & 3 à $18[12,14,15]$ \\
Sélénium & $58[11]$ \\
Zinc & 25 à $74[12,15]$ \\
Cuivre & $68[15]$ \\
Magnésium & $5[12]$ \\
Albumine & 0 à $13[12,14,15]$ \\
\hline
\end{tabular}


Tableau 2

Bilan nutritionnel préopératoire de patients (hommes et femmes) avec obésité morbide avant chirurgie bariatrique au CHU de Liège (moyenne \pm DS).

\begin{tabular}{|c|c|c|}
\hline $\begin{array}{l}\text { IMC : } \\
42 \pm 5 \mathrm{~kg} / \mathrm{m}^{2}\end{array}$ & $\begin{array}{l}\text { Hommes }(n=36) \\
\text { Valeurs (normes) } \\
\% \text { de sujets déficients }\end{array}$ & $\begin{array}{l}\text { Femmes }(n=67) \\
\text { Valeurs (normes) } \\
\% \text { de sujets déficients }\end{array}$ \\
\hline $\begin{array}{l}\text { Hémoglobine } \\
(\mathrm{g} / \mathrm{dL})\end{array}$ & $\begin{array}{l}15,3 \pm 1,0(13,3-17,2) \\
0 \%\end{array}$ & $\begin{array}{l}13,5 \pm 0,9(11,7-14,9) \\
7,5 \%\end{array}$ \\
\hline $\begin{array}{l}\text { Ferritine } \\
\qquad(\mathrm{ng} / \mathrm{mL})\end{array}$ & $\begin{array}{l}239 \pm 167(30-400) \\
0 \%\end{array}$ & $\begin{array}{l}99 \pm 95(15-400) \\
12 \%\end{array}$ \\
\hline $\begin{array}{l}\text { Acide folique } \\
\text { érythrocytaire } \\
\text { (ng/mL) }\end{array}$ & $\begin{array}{l}599 \pm 137(440-1140) \\
0 \%\end{array}$ & $\begin{array}{l}598 \pm 230(440-1140) \\
14 \%\end{array}$ \\
\hline $\begin{array}{l}\text { Vitamine B12 } \\
\quad(\mathrm{pg} / \mathrm{mL})\end{array}$ & $\begin{array}{l}364 \pm 112(>200) \\
3 \%\end{array}$ & $\begin{array}{l}329 \pm 116(>200) \\
9 \%\end{array}$ \\
\hline $\begin{array}{l}\text { Calcium } \\
\quad(\mathrm{mmol} / \mathrm{L})\end{array}$ & $\begin{array}{l}2,34 \pm 0,09(2,15-2,6) \\
3 \%\end{array}$ & $\begin{array}{l}2,27 \pm 0,08(2,15-2,6) \\
3 \%\end{array}$ \\
\hline $\begin{array}{l}\text { 25-OH vitamine } \\
\mathrm{D}_{3} \\
(\mathrm{ng} / \mathrm{mL})\end{array}$ & $\begin{array}{l}17 \pm 8(>32) \\
100 \%\end{array}$ & $\begin{array}{l}18 \pm 9(>32) \\
88 \%\end{array}$ \\
\hline $\begin{array}{l}\text { Magnésium } \\
(\mathrm{mmol} / \mathrm{L})\end{array}$ & $\begin{array}{l}0,84 \pm 0,07 \\
(0,75-1,00) \\
15 \%\end{array}$ & $\begin{array}{l}0,80 \pm 0,08 \\
(0,75-1,00) \\
7 \%\end{array}$ \\
\hline $\begin{array}{l}\text { Albumine } \\
\text { (g/L) }\end{array}$ & $\begin{array}{l}45 \pm 2,8(38-49) \\
0 \%\end{array}$ & $\begin{array}{l}42 \pm 3,1(38-49) \\
33 \%\end{array}$ \\
\hline $\begin{array}{l}\text { CRP } \\
\qquad(\mathrm{mg} / \mathrm{L})\end{array}$ & $\begin{array}{l}5,8 \pm 4,4(0-6) \\
25 \%\end{array}$ & $\begin{array}{l}10,5 \pm 10(0-6) \\
61 \%\end{array}$ \\
\hline
\end{tabular}

résultats assez comparables à ceux rapportés ci-dessus (carence en acide folique, en vitamine B12, en vitamine D). Toutefois, nos résultats mettent en évidence une déficience variable en fonction du sexe, en particulier pour l'acide folique, l'hémoglobine et la ferritine. La valeur de la CRP est également accrue dans notre bilan préopératoire, confirmant l'inflammation de bas grade décrite en relation avec l'obésité [16], en particulier chez les femmes, ce qui a déjà été rapporté par d'autres [17]. Un suivi postopératoire des différents paramètres rapportés dans le Tableau 2 est en cours.

\section{2. Étiologies des carences en micronutriments chez le sujet obèse et conséquences}

Les concentrations abaissées en micronutriments chez le sujet présentant une obésité morbide peuvent être attribuées à différents mécanismes. Néanmoins, la cause dominante apparaît d'ordre hygiéno-diététique [13]. Un apport faible en fruits et en légumes, ainsi qu'une consommation élevée en sucres raffinés, lipides et alcool, est habituellement rapportée dans l'obésité sévère [18]. Le tabagisme, qui reste plus fréquent chez les sujets obèses, peut expliquer en partie la concentration plasmatique abaissée en acide ascorbique [19]. La composition corporelle peut également influencer le statut vitaminique ; une concentration plasmatique basse en vitamine $\mathrm{C}$ est en relation avec une disposition centrale de l'adiposité [20], tandis qu'une concentration abaissée en $25-\mathrm{OH}$ vitamine $\mathrm{D}$ peut être liée à une rétention dans le tissu adipeux [21]. Différentes études observationnelles menées dans la population générale ont montré une association entre le niveau en micronutriments, en particulier vitaminique, et différentes morbidités, au-delà des pathologies habituellement rapportées avec des déficits avérés en micronutriments. Par exemple, des concentrations abaissées en vitamines B6 sont en relation avec une symptomatologie dépressive [22], un risque accru d'accident vasculaire cérébral [23] et le cancer colorectal [24]. Une concentration plasmatique abaissée en vitamine $C$ est associée avec un accroissement de la mortalité [25] et un risque accru d'infarctus du myocarde [26]. Une association inverse entre les concentrations plasmatiques en $25-\mathrm{OH}$ vitamine $\mathrm{D}$ et l'amplitude de l'insulinorésistance [13], mais également l'incidence du diabète [27] a aussi été rapportée.

\subsection{Recommandations}

À ce jour, il n'existe aucune étude d'intervention publiée rapportant les résultats d'une supplémentation visant à corriger une déficience nutritionnelle en micronutriments chez le sujet obèse avant chirurgie bariatrique. Ce type d'étude est bien entendu nécessaire mais actuellement, il n'est donc pas possible d'affirmer qu'une supplémentation nutritionnelle préopératoire permettrait de réduire les carences postopératoires observées, ni influencerait à terme la morbi-mortalité des sujets obèses opérés. Il existe néanmoins des recommandations publiées par différentes sociétés savantes portant sur la prise en charge nutritionnelle des sujets obèses en période préopératoire. Aux États-Unis, différentes sociétés savantes médico-chirurgicales impliquées dans la prise en charge des sujets obèses ont publié des recommandations communes [28]. Ces auteurs recommandent que tous les sujets obèses doivent a priori bénéficier en préopératoire d'une évaluation complète (anamnèse, examens clinique et biologique) (recommandation de grade A). Concernant les micronutriments en particulier, une évaluation sélective devrait être réalisée avant toute chirurgie bariatrique (recommandation de grade C), sans toutefois préciser ce qu'il faut doser spécifiquement. Finalement, ces auteurs recommandent, compte tenu du risque élevé de développement de déficience postopératoire après des procédures induisant une malabsorption, que le bilan préopératoire comprenne au minimum un bilan martial (fer sérique, ferritine), un dosage de la vitamine B12 et de la $25-\mathrm{OH}$ vitamine $\mathrm{D}$. Un traitement pour toute déficience significative, comme une anémie ferriprive, doit être initié en préopératoire.

En 2010, la Société francophone de nutrition clinique et métabolisme (SFNEP) et la Société française d'anesthésie et réanimation (SFAR) ont publié conjointement des recommandations de bonnes pratiques cliniques sur la nutrition périopératoire [29]. Les auteurs soulignent qu'un sujet obèse est potentiellement dénutri et qu'une perte de poids préopératoire constitue un facteur de risque indépendant de complication. Pour le sujet âgé, il faut assurer en préopératoire un apport en protéines de 1,2 à $1,5 \mathrm{~g} / \mathrm{kg}$ par jour. Ils confirment par ailleurs que le risque de carences spécifiques en micronutriments (vitamines, oligoéléments) est plus élevé chez le sujet obèse que chez le sujet de poids normal. En particulier, ils insistent, à l'instar de leurs collègues américains, sur la prévalence élevée de la carence en fer et 
sur l'importance d'un dépistage préopératoire systématique (fer sérique et ferritine) avec correction le cas échéant. Les auteurs proposent également des suppléments de thiamine (vitamine B1) en cas de perfusion de sérum glucosé ou de troubles digestifs. Concernant la vitamine B12, une supplémentation préopératoire est probablement nécessaire. Enfin, en cas d'amaigrissement préopératoire, un bilan nutritionnel plus complet est souhaitable avec dosage des vitamines B1, B9 (acide folique), B12, C, A, D et E.

Une autre question régulièrement posée concerne l'intérêt d'un amaigrissement volontaire chez le sujet obèse, en particulier avant chirurgie bariatrique. Pour les auteurs du consensus français, cet amaigrissement est inutile car il n'influence ni la mortalité, ni l'incidence des complications postopératoires, ni la perte de poids à long terme [29]. Cependant, deux études publiées récemment vont un peu à l'encontre de ces recommandations et préconisent au contraire un amaigrissement préopératoire chez le sujet obèse avant chirurgie bariatrique, même si les résultats objectifs de la perte de poids préopératoire (réduction de la perception de la difficulté de la procédure chirurgicale) demeurent modestes [30,31]. Le débat reste donc ouvert à l'heure actuelle.

\section{Conclusions}

Bien que les sujets avec obésité morbide aient un apport énergétique supérieur aux sujets non obèses, il peut exister chez les sujets obèses des carences nutritionnelles, en particulier en micronutriments. Or, il est bien établi que la chirurgie bariatrique s'accompagne de carences nutritionnelles qui pourraient donc être, en partie du moins, la conséquence de carences préopératoires. La cause principale de ces carences est liée à une alimentation riche en aliments à forte densité énergétique, mais pauvre sur le plan nutritionnel, notamment au niveau de l'apport en vitamines et oligo-éléments. La biodisponibilité de ces micronutriments pourrait également être réduite chez le sujet obèse. Les micronutriments les plus souvent concernés par les déficiences et la prévalence exacte de celles-ci restent mal documentés; enfin, même si certaines associations entre déficiences en micronutriments et risque accru de différentes pathologies (diabète, pathologies cardiovasculaires, dépression) ont été rapportées, les conséquences cliniques exactes de ces déficiences a minima demeurent très mal connues. Chez les sujets avec obésité morbide qui vont bénéficier d'une chirurgie bariatrique, un bilan nutritionnel préopératoire pourrait être envisagé, mais ce dernier reste mal systématisé, en dépit de recommandations récentes. Enfin, l'intérêt d'une supplémentation nutritionnelle préopératoire reste également à déterminer. Cependant, la solution à ces carences nutritionnelles ne passe probablement pas par la prescription de nombreuses vitamines et autres compléments alimentaires, mais bien par une alimentation saine qui contient tous les éléments nécessaires.

\section{Déclaration d'intérêts}

Les auteurs déclarent ne pas avoir de conflits d'intérêts en relation avec cet article.

\section{Références}

[1] Karlsson J, Taft C, Sjöström L, Torgerson JS, Sullivan M. Psychosocial functioning in the obese before and after weight reduction: construct validity and responsiveness of the obesity-related problems scale. Int J Obes Relat Metab Disord 2003;27:617-30.

[2] McTigue K, Larson JC, Valoski A, Burke G, Kotchen J, Lewis CE, et al. Mortality and cardiac and vascular outcomes in extremely obese women. JAMA 2006;296:79-86.

[3] Adams KF, Schatzkin A, Harris TB, Kipnis V, Mouw T, Ballard-Barbash $\mathrm{R}$, et al. Overweight, obesity, and mortality in a large prospective cohort of persons 50 to 71 years old. N Engl J Med 2006;355:763-78.

[4] Prospective studies collaboration. Body mass index and cause-specific mortality in 900,000 adults: collaborative analyses of 57 prospective studies. Lancet 2009;373:1083-96.

[5] Sturm R. Increases in morbid obesity in the USA: 2000-2005. Public Health 2007;121:492-6.

[6] Karlsson J, Taft C, Ryden A, Sjöström L, Sullivan M. Ten-year trends in health-related quality of life after surgical and conventional treatment for severe obesity: the SOS intervention study. Int J Obes (Lond) 2007;32:1248-61.

[7] Sjöström L, Narbro K, Sjöström CD, Karason K, Larsson B, Wedel H, et al. Effects of bariatric surgery on mortality in Swedish obese subjects. N Engl J Med 2007;357:741-52.

[8] Sjöström L, Peltonen M, Jacobson P, Sjöström CD, Karason K, Wedel $\mathrm{H}$, et al. Bariatric surgery and long-term cardiovascular events. JAMA 2012;307:56-65.

[9] Ziegler O, Sirveaux MA, Brunaud L, Reibel N, Quilliot D. Medical follow-up after bariatric surgery: nutritional and drug issues. General recommendations for the prevention and treatment of nutritional deficiencies. Diabetes Metab 2009;35:544-57.

[10] Kaidar-Person O, Person B, Szomstein S, Rosenthal RJ. Nutritional deficiencies in morbidly obese patients: a new form of malnutrition? Part A: vitamins. Obes Surg 2008;18:870-6.

[11] Kaidar-Person O, Person B, Szomstein S, Rosenthal RJ. Nutritional deficiencies in morbidly obese patients: a new form of malnutrition? Part B: minerals. Obes Surg 2008;18:1020-34.

[12] Ernst B, Thurnheer M, Schmid S, Schultes B. Evidence for the necessity to systematically assess micronutriment status prior to bariatric surgery. Obes Surg 2009;19:66-73.

[13] Aashein ET, Høfso D, Hjelmesøœth J, Birkeland KI, Bøhmer T. Vitamin status in morbidly obese patients: a cross-sectional study. Am J Clin Nutr 2008;87:362-9.

[14] Schweiger C, Weiss R, Berry E, Keidar A. Nutritional deficiences in bariatric surgery candidates. Obes Surg 2010;20:193-7.

[15] De Luis DA, Pacheco D, Izaola O, Terroba MC, Cuellar L, Cabezas G. Micronutrient status in morbidly obese women before bariatric surgery. Surg Obes Relat Dis 2012, in press, [Available online 29 September 2011].

[16] Lumeng CN, Saltiel AR. Inflammatory links between obesity and metabolic disease. J Clin Invest 2011;121:2111-7.

[17] Ford ES. Body mass index, diabetes and C-reactive protein among U.S. adults. Diabetes Care 1999;22:1971-7.

[18] Lissner L, Lindroos AK, Sjöström L. Swedish obese subjects (SOS): an obesity intervention study with a nutritional perspective. Eur J Clin Nutr 1998;52:316-22.

[19] Galan P, Viteri FE, Bertrais S, Czernichow S, Faure H, Arnaud J, et al. serum concentrations of beta-carotene, vitamins $\mathrm{C}$ and $\mathrm{E}$, zinc and selenium are influenced by sex, age, diet, smoking status, alcohol consumption and corpulence in a general French adult population. Eur J Clin Nutr 2005;59:1181-90

[20] Canoy D, Wareham N, Welch A, Bingham S, Luben R, Day N, et al. Plasma ascorbic acid concentrations and fat distribution in 19,068 British men and women in the European prospective investigation into cancer and nutrition Norfolk cohort study. Am J Clin Nutr 2005;82:1203-9.

[21] Wortsman J, Matsuoka LY, Chen TC, Lu Z, Holick MF. Decreased bioavailability of vitamin D in obesity. Am J Clin Nutr 2000;72:690-3.

[22] Hvas AM, Juul S, Bech P, Nexo E. Vitamin B6 level is associated with symptoms of depression. Psychother Psychosom 2004;73:340-3. 
[23] Kelly PJ, Shih VE, Kistler JP, Barron M, Lee H, Mandell R, et al. Low vitamin B6 but not homocyst(e)ine is associated with increased risk of stroke and transient ischemic attack in the era of folic acid grain fortification. Stroke 2003;34:51e-4e.

[24] Wei EK, Giovannucci E, Selhub J, Fuchs CS, Hankinson SE, Ma J. Plasma vitamin B6 and the risk of colorectal cancer and adenoma in women. J Natl Cancer Inst 2005;97:684-92.

[25] Khaw KT, Bingham S, Welch A, Luben R, Wareham N, Oakes S, et al. Relation between plasma ascorbic acid and mortality in men and women in EPIC-Norfolk prospective study: a prospective population study. European prospective investigation into cancer and nutrition. Lancet 2001;357:657-63.

[26] Nyyssonen K, Parviainen MT, Salonen R, Tuomilehto J, Salonen JT. Vitamin $\mathrm{C}$ deficiency and risk of myocardial infarction: prospective population study of men from eastern Finland. BMJ 1997;314:634-8.

[27] Scragg R, Sowers M, Bell C. Serum 25-hydroxyvitamin D, diabetes, and ethnicity in the third national health and nutrition examination survey. Diabetes Care 2004;27:2813-8.
[28] Mechanick JI, Kushner RF, Sugerman HJ, Gonzalez-Campoy JM, CollazoClavell ML, Spitz AF, et al. AACE/TOS/ASMBS guidelines. Guidelines for clinical practice for the perioperative nutritional, metabolic, and nonsurgical support of the bariatric surgery patient. Sur Obes Relat Dis 2008;4:S109-84.

[29] Chambrier C, Sztark F. Recommandations de bonnes pratiques cliniques sur la nutrition périopératoire. Actualisation 2010 de la conférence de consensus de 1994 sur la «nutrition artificielle périopératoire en chirurgie programmée de l'adulte». Nutr Clin Metab 2011;25: 48-56.

[30] Cassle S, Menezes C, Birch DW, Shi X, Karmali S. Effect of preoperative weight loss in bariatric surgical patients: a systematic review. Surg Obes Relat Dis 2011;7:760-7.

[31] Van Nieuwenhove Y, Dambrauskas Z, Campillo-Soto A, van Dielen F, Wiezer R, Jansen I, et al. Preoperative very low-calorie diet and operative outcome after laparoscopic gastric bypass: a randomized multicenter study. Arch Surg 2011;146:1300-5. 